\title{
Airborne Doppler LiDAR Sensor Parameter Analysis for Wake Vortex Impact Alleviation Purposes
}

\author{
Jana Ehlers and Nicolas Fezans \\ Citation Info: \\ Ehlers, Jana and Fezans, Nicolas (2015) Airborne Doppler LiDAR Sensor Parameter Analysis for \\ Wake Vortex Impact Alleviation Purposes. In Book: Selected Papers of the 3rd CEAS Specialist \\ Conference on Guidance, Navigation and Control, pp. 433-453. Springer-Verlag Berlin Heidelberg. \\ CEAS EuroGNC Conference, 13.-15. April 2011, Toulouse, France. ISBN 978-3-319-17517-1.
}

\begin{abstract}
This paper presents a sensitivity study of a wake vortex impact alleviation system based on an airborne forward-looking Doppler LiDAR sensor. The basic principle of the system is to use this sensor to measure the wind remotely ahead of the aircraft. On the basis of these measurements the system estimates whether a wake vortex is located in front of the aircraft. If this is the case, the wake vortex characteristics are identified and the control deflections countervailing the wake-induced aircraft response are computed and applied. An integrated simulation environment comprising a full nonlinear 6-DoF A320 model (with control laws), wake vortex models, and the wake impact alleviation algorithms was developed. The LiDAR sensor subsystem has many design parameters that influence the overall performance in a complex way, which makes it difficult to derive adequate requirements. The presented parameter study provides first insights into the role of each parameter as well as some adequate parameter combinations.
\end{abstract}

\section{Introduction}

Wake vortices are an inevitable phenomenon in air traffic. They evolve from the pressure difference between the lower and upper side of the wing during the lift generation and roll up to form a pair of two strong rotating flow fields. For an aircraft flying into these rotating flow fields, the wake vortices can pose a serious safety threat and can lead to dangerous aircraft reactions usually involving a sudden rolling motion or a loss of altitude. As wake vortices are under most atmospheric conditions not visible the aircraft reaction occurs unexpectedly for the pilot and increases his workload. In extreme cases a wake vortex encounter can cause structural damages to the aircraft, lead to incidents with injuries of the passengers and crew [1-3] or even a crash of the aircraft [4,5]. In order to prevent the encoun-

\author{
Jana Ehlers \\ DLR (German Aerospace Center), Institute of Flight Systems, Lilienthalplatz 7, 38108 Braun- \\ schweig, Germany, Jana.Ehlers@dlr.de \\ Nicolas Fezans \\ DLR (German Aerospace Center), Institute of Flight Systems, Lilienthalplatz 7, 38108 Braun- \\ schweig, Germany, Nicolas.Fezans@dlr.de
}


ter of dangerous wake vortices, ICAO introduced separation minima for approach and departure. However, the drawback of this safety measure is the resulting air traffic capacity limitation. Finding ways to reduce these separation requirements while at least maintaining the same safety standards will certainly bring significant benefits for congested airports.

A possible approach to achieve this is to reduce the impact of a wake vortex on the encountering aircraft by equipping this aircraft with a specific control system for wake vortex encounters. Looye et al. [6] and Rafi and Steck [7], included wake vortices in their control design. They considered them as one of the disturbances the flight controller should be able to handle. Another approach is a control system which is based on a remote wind sensor [8-10].

The generic term "remote wind sensor" is chosen because different sensor types could be used. It could for instance be a so-called Doppler LiDAR sensor. Doppler LiDAR sensors permit to measure one component of the wind at a remote location. A LASER source is used to illuminate the location at which the measurement shall be made. At this location, particles (e.g. aerosols or even molecules) are scattering this light back to the sensor which also contains a detector. The backscattered light properties provide information on the corresponding particles. The present work only considers the relative wind velocity measurements that can be deduced from the Doppler frequency shift between the original LASER source and the received backscattered light. The Doppler frequency shift is proportional to the rate of change of the distance between the sensor and the particles, which means that the relative velocity component that is measured is the component that is in the direction of the line joining the sensor to the particles location (assuming that the light source and the detector are colocated). This direction is commonly called "Line-of-Sight" (LoS). The amount of light that is backscattered decreases with the square of the measurement distance and consequently the measurement uncertainty increases correspondingly.

The location where the measurement is made depends on the time elapsed between the moment the light was emitted and the moment at which the received backscattered light was observed by the detector. Indeed, both the pulse and the observation typically last for a few tenths of nanoseconds, which implies that the measured location is not a point but rather has a very long frustoconical shape. As the beam divergence is usually very low, an approximation of this domain with a cylinder or even with a line can usually be made. The resulting averaging effect that results from not measuring in only one point is often referred by the term blur, by analogy with photo imaging.

The investigations of [8-10] were based on the assumption that sensors capable of measuring the wind in 3D would be available. It seems that LoS-only Doppler LiDAR might be available for airborne application in the near future, whereas 3D remote wind sensors would probably remain too experimental and costly in the foreseeable future. Even when only considering LoS velocity measurements, there are plenty of choices to be made for the technical characteristics of the Doppler LiDAR sensors: wavelength, type of pulses (shape, duration), LoS directions 
(scanning geometry, field-of-view), number of domains being measured along the LASER beam line, detector technologies, etc. These parameters influence the measurement quality (e.g. noise and precision) and/or the collected information itself (spatial resolution, blurring effect, measurement location). The present study aims at helping to find a good trade-off between the possible Doppler LiDAR configurations by considering the aircraft dynamics as well as the flight control system and the whole sensor measurement processing chain.

When measuring the wind only in LoS direction, two of the three components are necessarily missing and from a flight control point of view, the remaining LoS direction usually contains only marginal part of the relevant information. In [11] the first author of the present paper and two colleagues proposed a solution to this problem that was called "Wake Impact Alleviation Control" (WIAC). This system consists of two main steps. First an Online Wake Identification (OWI) algorithm is used to reconstruct the wake vortex disturbance from several LoS measurements. Then the identified wake vortex model is used to predict the controldeflection-free wake-induced aircraft response that the control system countervails by commanding the adequate control surface deflections. This wake impact alleviation concept was found very promising [11] based on a simplified simulation setup. The wake impact alleviation control system was applied in a complete 6-DoF simulation environment wherein the aircraft encounters a wake vortex. However, in the simulations of [11] the online wake identification algorithm was not coupled with the 6-DoF simulation, but had been performed beforehand in a separate simplified 3-DoF simulation of the same encounter whereupon the wake vortex disturbance did not cause any aircraft reaction. Consequently, the influence of the aircraft reaction on the measurement of the Doppler LiDAR sensor was neglected. Additionally, once a wake vortex pair matching well the measurements was found, this solution was kept and not updated.

The current paper presents the fully integrated wake identification and impact alleviation control system OWIDIA, which consists of OWI and WIAC. An extensive parameter study was performed during summer 2014 with a total of 18432 full 6-DoF simulations with online wake identification performed every $200 \mathrm{~ms}$ (simulation time) using a small-scale grid computer (up to 16 simulations in parallel). Some of the first insights gained from these data on the performance are presented in this paper and will serve to improve the current design of the whole system.

\section{Wake Impact Alleviation Approach}

The concept of the wake impact alleviation approach considered in this paper is shown in Fig. 1. The overall principle is identical to the approach presented in [11]. The main difference consists in the implementation of the online wake identification, which is now fully integrated into the control system. The purpose of 
the control system is to compensate the disturbance wind velocities of the wake vortex by specific control command deflections which prevent a wake-induced aircraft reaction. The disturbance wind velocities and the commanded control surface deflections of the WIAC consequently represent the input and output of the complete process as illustrated in Fig. 1.

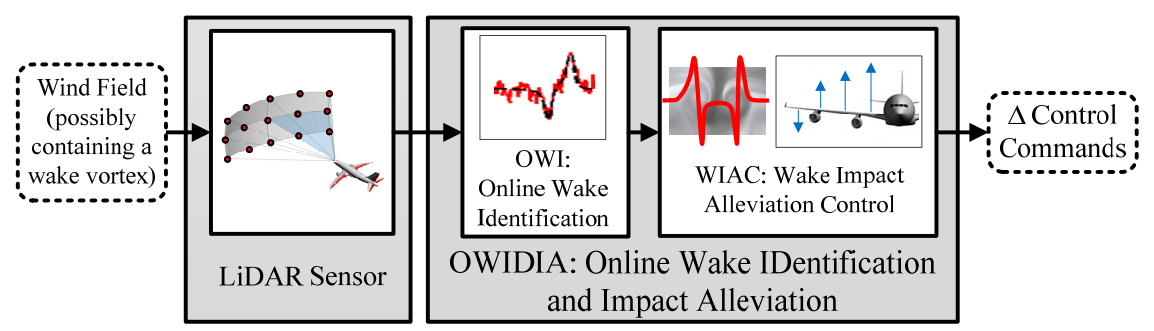

Fig. 1 Concept for wake impact alleviation

The LiDAR sensor measures the wind velocities at several locations within a relatively short range ahead of the aircraft. As already mentioned, each measurement is a line-of-sight velocity, which is then passed to the online wake identification algorithm. The OWI identifies a wake vortex model which provides the best match with the provided line-of-sight measurements and hands the parameters of this model to the wake impact alleviation control module. This module derives the wake induced aircraft disturbance on the basis of the identified wake model and calculates the required control surface deflection to countervail the wake-induced aircraft response. Further details on the different elements of the OWIDIA system are given in the following.

\subsection{Online Wake Identification}

The LiDAR sensor only provides LoS measurements, in which most of the information about the wake vortex wind velocities is lost. The online wake identification needs to reconstruct both the two lost components of the wind measurements and the wind at the locations where no measurement was made. The principle of the online wake identification is adapted from [12,13] and was also described in [11]. For the integrated implementation in the OWIDIA system within the full 6DoF simulation framework, some extensions and modifications of the OWI were realized. Fig. 2 gives an overview over the new process of the OWI and its integration into the flight control.

The OWI is usually called with a lower update rate than the LiDAR sensor and the rest of the flight control system. The measurements of the sensor over a time frame of 2 seconds are stored in a measurement buffer. Depending on the sensor characteristics this can be one or several measurements at a time. The location of 
each measurement is stored in an airframe-carried north-east-down (NED) coordinate system. As the aircraft moves the coordinates of each measurement relative to the aircraft are updated in the measurement buffer. The software modules that are managing the measurement buffer and computing the control commands both run at every major step of the simulation (i.e. with $100 \mathrm{~Hz}$ in the applied simulation environment). Both modules should also provide a very similar performance if they were executed with larger sampling time. The OWI is executed with a lower frequency because it is assumed that it requires a larger computation time. For the studies presented here we chose an update rate of $5 \mathrm{~Hz}$ for the OWI because this would correspond to a realistic trade-off between responsiveness to new measurements and available computation time. Reaching hard real-time requirements for an update rate of $5 \mathrm{~Hz}$ seems achievable, but is not a priority at the current stage of this work. The OWI update rate can be varied independently from the update rate of the LiDAR measurements as these are buffered beforehand.

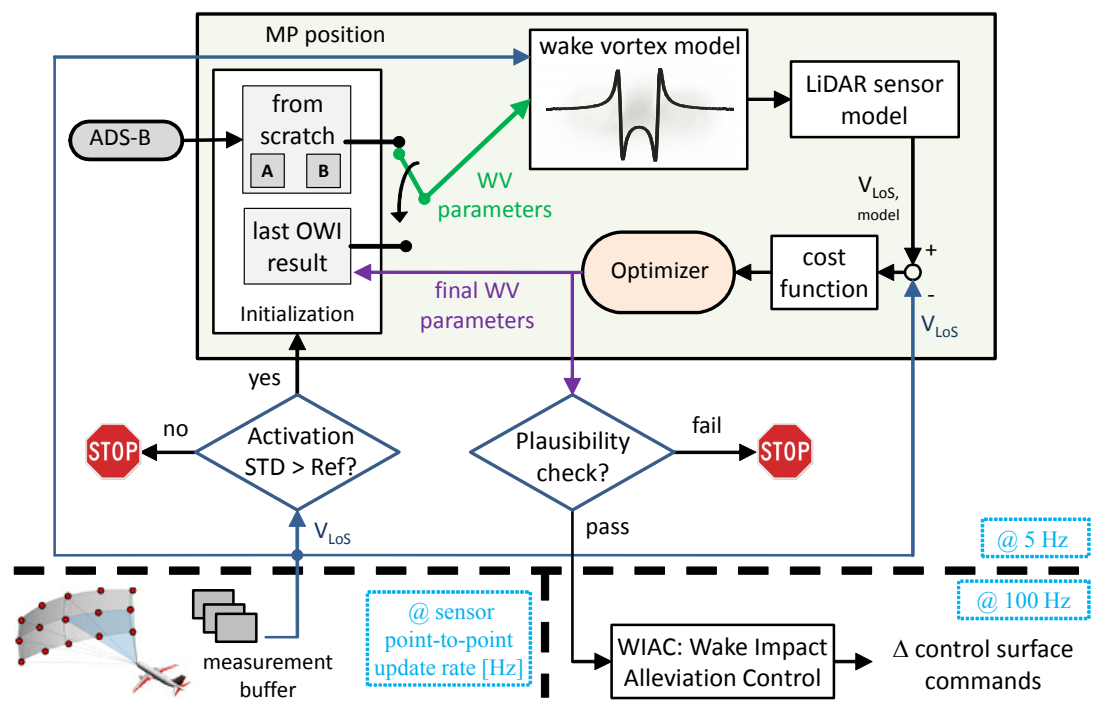

Fig. 2 Online wake identification workflow

Every time the OWI is called, first a very simple criterion tries to determine if there are strong indications of a possible wake vortex in the current content of the measurement buffer. This permits to avoid an execution of the OWI if no wake vortex is present, which is the case during most of the flight time. It, thus, represents an additional safety net for most irrelevant situations (no wake vortex) and saved computing time during the parameter study. This very simple criterion is based on the comparison of the standard deviation of all line-of-sight measurements stored in the data buffer with the reference measurement noise. The identification algorithm in the OWI is only started if the current standard deviation of the LoS measurements in the buffer is larger than $120 \%$ of the reference value. If the standard deviation of the sensor measurement noise is reliably known this val- 
ue can be used directly as a reference value. There are also possibilities to estimate it online. In the current implementation the reference standard deviation of the LoS measurement is defined as the standard deviation at the beginning of the simulation right after the measurement buffer has been filled for the first time. The simulation is set up such that there are no significant wake-induced velocity measurements contained in the buffer at that time. The standard deviation of the LoS measurements thus corresponds approximately to the measurement noise.

Fig. 3 exemplarily shows a time history of the standard deviation of the line-ofsight wind velocities stored in the measurement buffer for a $30^{\circ}$ lateral encounter. When the data buffer is filled for the first time the standard deviation of the stored $\operatorname{LoS}$ velocities is $0.87 \mathrm{~m} / \mathrm{s}$. When no wake vortex is present the standard deviation keeps similar values. Between $16 \mathrm{~s}$ and $20 \mathrm{~s}$ the standard deviation rises significantly due to the wind velocities caused by the wake vortex and contained in the measurement buffer at that time. The LoS velocity criterion is activated between $16.5 \mathrm{~s}$ and $19.7 \mathrm{~s}$ (black solid line

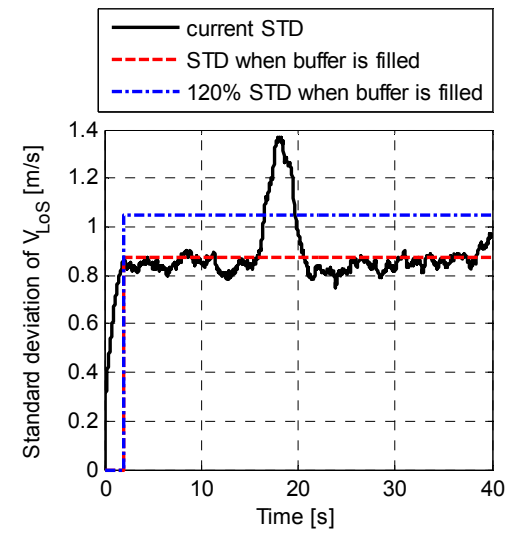

Fig. $3 \mathrm{LoS}$ velocity trigger for a $30^{\circ}$ lateral encounter above the dotted-dashed blue line in Fig. 3). When this happens the OWI algorithm starts.

This very simple criterion is such that the actual signal (wake vortex signature in the measurements) should cause an increase of at least $20 \%$ compared to the pure noise level. Such a criterion is certainly too restrictive for cases with relatively low signal-to-noise-ratios (SNR). "Low amplitude" (i.e. low circulation) wake vortices are not relevant for the designed system. However it might also be interesting to consider sensors with high spatial resolution but high noise levels. It is clear that the criterion used during this study is too simple to handle this type of cases and that it will have to be improved to investigate these cases, too.

The OWI problem is a maximum-likelihood estimation problem, which consists in finding parameter values of a wake vortex model such that the LoS velocities derived from the identified wake vortex model and the measured LoS velocities show the best possible match. This maximum likelihood process is solved using an optimization algorithm as shown in Fig. 2. The wake vortex model used in the current work is the well-known Burnham-Hallock model [15]. A strong dependence of the results on the model used (among the classical ones) is not expected. The resulting model-based LoS velocities of all measurement point positions in the data buffer are then compared to the actual LoS velocities of the real measurement. 


\subsubsection{Optimization algorithm}

The maximum likelihood problem can be easily solved with many classical optimization algorithms. For more explanation of the way to formulate such maximum likelihood problems and to solve them, refer to [14]. In [11], for practical reasons, the measurements from different times were grouped for each LoS direction. In the present implementation, measurements were not grouped in any way and it can be shown that the maximum-likelihood cost function is equivalent to a weighted least square fitting problem in that case.

When dealing with data buffers not containing any vortex, the maximum likelihood problem is usually badly conditioned and the algorithms will need many small steps to finally end up with a solution where the identified vortex either has a very small circulation or is located well outside of the domain containing the measurements. Even if none of these solutions is an issue, it is considered safer to detect this situation before even starting the algorithm. This was the main motivation for introducing the aforementioned activation criterion for the estimation process.

The algorithm used here is a Broyden-Fletcher-Goldfarb-Shanno and the line search step is performed by a nonstandard algorithm that combines several ideas from the classical line search algorithms depending on the situation. As already mentioned most standard algorithms will converge to the right solution if enough information is contained in the buffer: the differences are mainly a question of execution speed and the currently used solution was not particularly optimized.

\subsubsection{Identified Parameters}

The parameters of the wake vortex model which are optimized by the OWI are:

- the vortex circulation $\Gamma$ (which defines the strength of the vortex),

- the distance between the two vortex cores $b^{\prime}$

- and four geometry parameters specifying location and orientation of the wake vortex with respect to the aircraft.

The geometry parameters of the wake vortex are displayed in Fig. 4 and 5. The wake vortex location is defined by means of two points on the vortex centerline. These points are described in a particular coordinate system which is called identification (ID) coordinate system here. The ID-coordinate system is located in the horizontal $x-y$-plane behind the aircraft and its $x$-directions points into the direction of the body-fixed $\mathrm{x}$-axis projected into the horizontal plane. The $\mathrm{y}$-axis points to the right of the aircraft and the z-axis points downwards. The befenit of using a coordinate system that is not attached to the wake is that it significantly reduces the number of coordinate transformations that must be repeatedly made during the wake estimation process. The coordinates of the LoS measurements in this coordinate system are fixed during the entire estimation process. 
In this coordinate system two points $P_{1}$ and $P_{2}$ are specified which define the location of the wake vortex. Point $\mathrm{P}_{1}$ is located on the vortex centerline at the position where the projections of the body-fixed $\mathrm{x}$-axis of the aircraft and the vortex centerline into the horizontal $x$-y-plane cross each other. The y-position of $\mathrm{P}_{1}$ is zero by definition. Point $\mathrm{P}_{2}$ is defined as the position of the vortex center line for $\mathrm{x}_{\mathrm{ID}}=0$. The coordinates $\mathrm{x}_{1}, \mathrm{z}_{1}, \mathrm{y}_{2}$ and $\mathrm{z}_{2}$ of the points $\mathrm{P}_{1}$ and $\mathrm{P}_{2}$ uniquely describe the location and orientation of the wake vortex and are used as identification parameters for the OWI in the current implementation.

In Fig. 4 and 5 it can be noticed that the origin of the ID-coordinate system is located a distance $\Delta \mathrm{x}$ behind the center of gravity of the aircraft. This shift of the origin with respect to the center of gravity was introduced because the parameterization chosen has a singularity when the vortex centerline goes

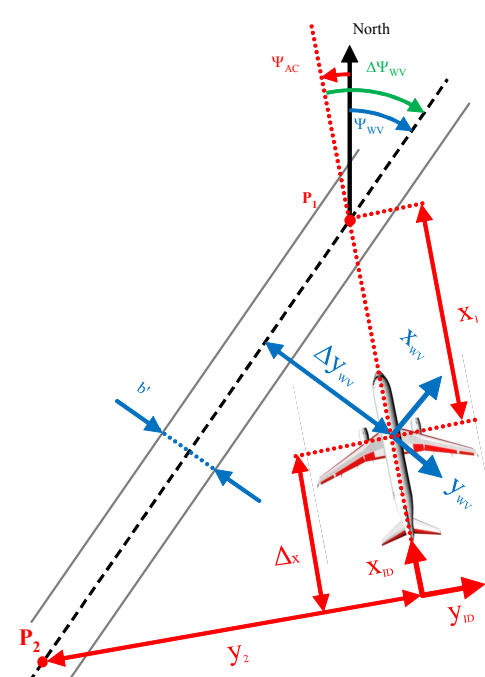

Fig. 4 Parameterization (top view)

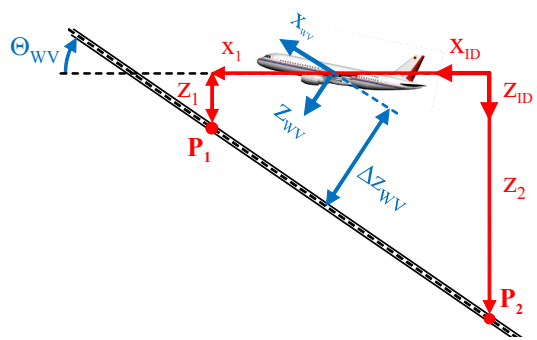

Fig. 5 Parameterization (side view) through the point $\left(\mathrm{x}_{\mathrm{ID}}, \mathrm{y}_{\mathrm{ID}}\right)=(0,0)$. By shifting the point $(0,0)$ far enough behind the aircraft, the corresponding vortex becomes practically uninteresting and irrelevant. In the current implementation the origin of the ID-coordinate system is located $200 \mathrm{~m}$ behind the center of gravity of the aircraft. Measurements made using a forward-looking sensor are basically around $\mathrm{P}_{1}$ (assuming there is a vortex to be detected), such that by varying $x_{1}$ and $z_{1}$ the position of the vortex within the measurement zone is varied, whereas a variation of $y_{2}$ and $z_{2}$ then lead to a change in orientation. The properties of the optimization problems with this choice of coordinate system and with the coordinates that were previously used in [11] were not compared.

\subsubsection{Initialization of the OWI Algorithm}

The OWI algorithm works iteratively and needs starting values for the identification parameters. Depending on whether the OWI has recently found a valid identification result or not these initial values are determined in two different ways. If 
the OWI has not identified a wake vortex shortly before, the initial parameters for the optimization routine are defined from scratch on the basis of the current aircraft dynamics and by means of input data from the generator aircraft which are available with standard Automatic Dependent Surveillance - Broadcast (ADS-B).

The initial values of the vortex circulation $\Gamma_{0}$ and the lateral vortex separation $\mathrm{b}_{0}^{\prime}$ are always set to $250 \mathrm{~m}^{2} / \mathrm{s}$ and $40 \mathrm{~m}$ for all wake encounter scenarios as suggested in [12]. An adequate initial value for parameter $\mathrm{x}_{1}$ corresponds to a distance that is slightly smaller than the distance to the farthest measurement point which is present in the buffer. To further increase the robustness for cases with no or only small influence of the wake vortex, the OWI is run with two different initial values for $\mathrm{x}_{1}$. For the second initial value of $\mathrm{x}_{1}$ a large distance of $500 \mathrm{~m}$ is added to the first initial value of $x_{1}$. This helps to detect the absence of a wake vortex because the second initial value of $x_{1}$ corresponds to a remote location of the wake vortex, which has practically no influence on the measurements. The corresponding OWI result has a lower cost function value and is thus selected as OWI output.

The initial value of $z_{1}$ is derived under the assumption that the point $P_{1}$ is located on the body-fixed $\mathrm{x}$-axis of the aircraft in the distance of the average measure-

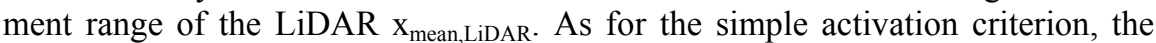
choices made for $\mathrm{x}_{1}$ and $\mathrm{z}_{1}$ should rather be seen as a first-shot but yet practical and functioning solution, which could relatively easily be improved and become more elegant. The basic idea behind these choices is to consider that if a vortex enters the measurement domain due to the aircraft translational motion, then this vortex is expected to be crossing the flight path of the aircraft approximately at the location defined by these initial $x_{1}$ and $z_{1}$.

The initial guess of the position of point $\mathrm{P}_{2}$ is determined by means of external input data via ADS-B. It is assumed that the generator aircraft is equipped with standard ADS-B and transmits its current azimuth and flight path angle. The initial values for the lateral and vertical position of point $\mathrm{P}_{2}$ can be derived easily from the position of $\mathrm{P}_{1}$ and these two angles.

These parameters are used as initial values for the optimization process if the OWI has not identified a wake vortex for a certain timeframe. If the OWI converged to a plausible result (cf. Section 2.1.4), the identified parameters are selected as initial values for the next OWI call. The only modification of the identified values of the last OWI call is the adaptation of the wake vortex position. As the OWI is called with a relatively low update rate, the aircraft has moved significantly when the optimization is started again. For an aircraft flying with $150 \mathrm{kt}$ during approach and an OWI call rate of $5 \mathrm{~Hz}$, as it is implemented for the studies presented here, the aircraft moves approximately $15 \mathrm{~m}$ between two OWI calls. The identified wake vortex position of the previous OWI call is thus corrected by the aircraft motion since this OWI call. The translation made between two successive OWI executions plays the exact same role as the "propagation steps" of Kalman or particle filters.

In case of using the last OWI result as an initial guess for the next OWI call it is not necessary to start the OWI with two different initial values. Only one optimization procedure is executed on the basis of the previously identified wake vortex. While the aircraft is encountering the same wake vortex, the initial parameters 
based on the last OWI result usually represent a better initial guess than estimated values on the basis of ADS-B data. After the aircraft has passed the wake vortex, however, it might encounter the wake generated by another aircraft. To get appropriate initial values for a potential new encounter the identified parameters are not used for the initialization anymore, if the OWI result has not been updated for 4 seconds. In this case it is assumed that the aircraft has passed the wake vortex and the initial OWI parameters are initialized from scratch with the help of ADS-B data again (cf. Fig. 2).

\subsubsection{Plausibility Check of Identified Wake Vortex}

After having identified a wake vortex, the physical plausibility of the identified wake vortex is checked. Six criteria are applied to evaluate if the output of the OWI is physically plausible. This plausibility check adds a safety net between the identification process and the use of the identified vortex by the flight control system. The validity criteria concern the vortex circulation and separation as well as the position and orientation of the wake vortex. The wake elevation $\Theta_{\mathrm{Wv}}$ (i.e. inclination of the vortex centerline with the respect to the horizontal plane) and the wake azimuth $\Psi_{\mathrm{WV}}$ (i.e. orientation of the vortex centerline with respect to north) are derived from the coordinates $\mathrm{x}_{1}, \mathrm{z}_{1}, \mathrm{y}_{2}$ and $\mathrm{z}_{2}$. In detail the six validity criteria are the following:

\section{Limits for circulation: $100 \mathrm{~m}^{2} / \mathrm{s}<\Gamma<500 \mathrm{~m}^{2} / \mathrm{s}$}

The limits for the identified vortex circulation are specified as suggested in [12] in order to filter out results caused by atmospheric turbulence without the presence of wake vortices as well as unrealistically high results.

2. Limits for lateral vortex separation : $10 \mathbf{m}<\mathrm{b}^{\prime}<\mathbf{1 0 0} \mathbf{m}$

According to [12], the lower bound of $10 \mathrm{~m}$ is applied in order to filter out wakes of small aircraft or wakes with advanced decay. The upper bound of $100 \mathrm{~m}$ corresponds to the wingspan of aircraft at the upper limits of the "heavy" category with some extra margin for moderate Crow instability.

3. Limits for wake elevation: $-\mathbf{1 0}^{\circ}<\boldsymbol{\Theta}_{\mathrm{wV}}<20^{\circ}$

The lower bound of $-10^{\circ}$ for valid wake elevation is selected as the sum of the estimated maximum descent angles of the generator aircraft and the wake vortex as suggested in [12]. The upper bound is selected as $20^{\circ}$ according to the assumed maximum climb rate of the generator aircraft [12].

4. Limits for wake azimuth: $\left|\boldsymbol{\Psi}_{\mathrm{WV}}-\chi_{\mathrm{ADS}-\mathrm{B}}\right|<15^{\circ}$

It is assumed that the deviation of the actual wake azimuth from the course angle of the generator aircraft transmitted via ADS-B shall be smaller than $15^{\circ}$. Identified wake vortices with larger deviation from the generator flight path azimuth are thus defined as invalid.

5. Plausibility check for vertical position of wake vortex centerline

The vertical position of the wake vortex is verified if the vortex centerline plus a certain tolerance lies within the vertical range of the stored measurement lo- 
cations. The reason for the addition of a tolerance is that the wake vortex still has a significant effect at a certain distance above and below the vortex centerline. The tolerance is chosen as $75 \%$ of an assumed lateral vortex core distance of $50 \mathrm{~m}$, i.e. $37.5 \mathrm{~m}$.

\section{Plausibility check for lateral position of wake vortex centerline}

Concerning the lateral position of the identified wake vortex it is checked whether the projection of the centerline onto the horizontal plane intersects (with a tolerance of $37.5 \mathrm{~m}$ ) the domain that contains the measurements (projected as well). This domain was approximated by a rectangle that contains all the measurements.

If all of these criteria are fulfilled the identified wake vortex of the OWI is considered as valid and is provided to the wake impact alleviation. If no new OWI result is available the control command generation for the wake impact alleviation control is based on the last available valid OWI result.

\subsection{Wake Impact Alleviation Control Command Generation}

The control command generation of the wake impact alleviation control system works identically to the process described in [11]. An overview of the workflow is illustrated in Fig. 7.

As already mentioned the OWI output contains the information on the identified wake vortex which the wake impact alleviation must countervail. Each time the control commands are computed, the relative position of the wake (with respect to the aircraft) must be updated beforehand. To compute the wake-induced forces and moments an aerodynamic interaction model (AIM) is used. The aircraft is divided into strips for which the disturbance wind velocities are determined. However, in order to account for the time delay due to processing time and actuator delays, the positions at which the wind velocities are calculated, are not the current positions of the strips, but their estimated positions at the time "now + total time delay". The wind velocities at each strip are directly provided by a wake vortex model using the identified parameter values. Based on this information the AIM computes the additional forces and moments induced by the wake. A more detailed description about the AIM model and its validation is given in [16].

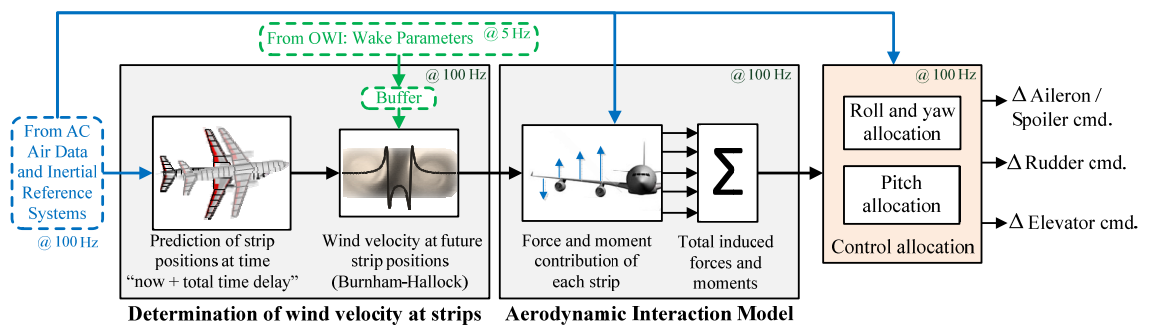

Fig. 7. Workflow of wake impact alleviation control command generation 
The goal of the wake impact alleviation control system is to compensate for these wake-induced forces and moments. Common aircraft configurations only use ailerons, elevators and rudder(s) as control surfaces with which only three degrees of freedom (roll, pitch and yaw) can be controlled. Consequently, a complete compensation of all six disturbance forces and moments is not achievable. Instead only the wake-induced moments are compensated by the WIAC to alleviate rotational aircraft movements resulting from the wake vortex. The required control surface deflections to counteract the wake-induced moments are determined by analytically inverting the aerodynamic control surface efficiency matrix of the aircraft. The determined control surface commands are added to the control surface deflections commanded by the regular flight control system.

\section{Assessment of Influence of LiDAR Sensor Characteristics on OWI and WIAC}

The presented approach for the alleviation of the wake-induced aircraft response obviously only works if the identified wake is sufficiently representative of the wind field ahead of the aircraft. From the best of the authors' knowledge, there is no commonly accepted criterion on which the definition of a required OWI performance could be based. In order to overcome this issue, a simplified sensitivity study was performed in [11], in which the WIAC performance for various wake parameter estimation errors was investigated. The identification of the parameters was not performed online, but "the estimation loop was opened" and the identified parameters were directly fed into the WIAC as constant input values. The simulations carried out in the present study were all performed with the wake identification in the closed-loop. The parameters whose sensitivities are being analyzed are the Doppler LiDAR parameters instead of the wake parameter estimation errors as in [11]. The goal of this study is to assess the performance of the whole OWIDIA system (OWI + WIAC) and thereby to represent a first major step towards identifying the best sensor parameter combinations (ultimately permitting to derive a complete set of requirements for the sensor specialists / manufacturers). This study shall also help detecting the limits of the system or potential problems that still need to be addressed.

\subsection{Setup of the Sensitivity Study}

The challenge of defining required LiDAR sensor characteristics results from the fact that a LiDAR sensor has a large number of different parameters whose effects are not independent from each other and strongly nonlinear. Many parameters of the Doppler LiDAR sensor influence the performance of the complete system. In 
order to limit the number of simulations to a reasonable number some parameters were not varied and for those that were varied only a limited number of values was considered.

In order to identify which of the various parameter combinations represent feasible and desirable LiDAR settings an automatized and distributed simulation framework has been set up, in which the LiDAR sensor parameters are systematically varied and combinations of ranges of different sensor parameters are studied for different encounter scenarios. The goal of the sensitivity study is to find the possible interesting sensor characteristics and not yet to make a thorough assessment of a given system configuration. Therefore, the WIAC is not considered for many different encounter scenarios with a given set of sensor characteristics, but rather for many sensor characteristics and a few encounter configurations. In all cases considered hereafter an Airbus A320 aircraft encounters the wake of an Airbus A340 aircraft during approach. The wake vortex is always located $2 \mathrm{~m}$ above the center of gravity of the encountering aircraft and the vertical encounter angle is $0^{\circ}$. The lateral encounter angle $\Delta \Psi_{\mathrm{WV}}$ is varied between $5^{\circ}, 10^{\circ}, 15^{\circ}$ and $30^{\circ}$. Larger encounter angles are not considered because the encounter time is too short to induce a critical aircraft reaction. The ranges of the LiDAR parameters considered in the sensitivity analysis are shown in Table 1 . Hereafter the location of each measurement is called measurement point (MP) and is defined as the middle point of the corresponding measurement volume.

Table 1 LiDAR parameters for sensitivity study

\begin{tabular}{|c|c|}
\hline Parameter & Range of values \\
\hline minimum measurement range range $e_{\min }[\mathrm{m}]$ & $60 ; 90 ; 120 ;(150)$ \\
\hline lateral Scan angle range $\Psi_{\text {scan }}\left[{ }^{\circ}\right]$ & $+/-16 ;+/-30 ;+/-40$ \\
\hline vertical Scan angle range $\Theta_{\text {scan }}\left[{ }^{\circ}\right]$ & $+/-10$ \\
\hline \# MP along measurement axis, $\mathrm{N}_{\mathrm{a}}(2$ in Fig. 8 ) & $1 ; 3 ; 5$ \\
\hline \# horizontal MP axes, $\mathrm{N}_{\mathrm{h}}(3$ in Fig. 8 ) & $3 ; 5 ; 7 ; 9$ \\
\hline blur depth [m] & $15 ; 30$ \\
\hline full screen update rate $[\mathrm{Hz}]$ & $5 ; 10$ \\
\hline
\end{tabular}

The parameters defining the geometry of the LiDAR measurement are shown in Fig. 8. The length of each measurement volume in axial direction is defined by the parameter blur depth. Along one measurement axis there can be more than one measurement point. The position of the measurement point located closest to the LiDAR sensor is described by the parameter range min $_{\text {. }}$.

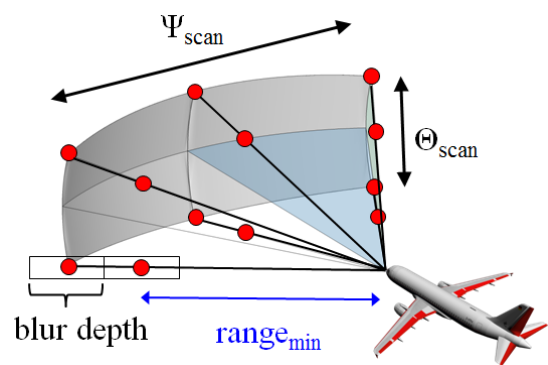

Fig. 8 Geometric LiDAR parameters 
Further measurement points are always located subsequently with a distance corresponding to the blur depth after the prior measurement point. The total number of measurement points in a full scan cycle results from the combination of the number of measurement points along each axis $\mathrm{N}_{\mathrm{a}}$ and the number of measurement axes. The number of measurement axes in horizontal direction $\mathrm{N}_{\mathrm{h}}$ is varied between the values shown in Table 1. In vertical direction, the number of 3 vertical axes is kept constant for all simulations. The field-of-view of the LiDAR sensor is defined by the maximum scan angles in lateral $\left(\Psi_{\text {scan }}\right)$ and vertical $\left(\Theta_{\text {scan }}\right)$ direction. The update rate of the measurements is defined by the "full screen update rate". This parameter specifies the frequency with which all measurement points in the scanning sphere are updated. Nevertheless, each measurement axis is updated subsequently. That means the point-to-point update rate varies if the number of measurement axes is varied. All measurement points along one axis are updated simultaneously.

The minimum range parameter in parentheses in Table 1 is not included in the results that are displayed in Section 3.2. For a minimum range of $150 \mathrm{~m}$ it turned out that the measurement noise, which increases with increasing measurement distance, is too high and the optimization algorithm has rarely been executed for almost all sensor characteristics.

The dependency of the measurement errors and noise levels on the Doppler LiDAR parameters is very difficult to model. The LiDAR model that is used in the simulation environment includes the main dependencies in a generic fashion. In addition to the parameters that are varied here further parameters such as the detector properties (e.g. resolution and sensitivity) and the LASER source properties (e.g. quality, output power) would also influence the measurement errors. Moreover the measurement errors, as considered in the current paper, are the errors on the determination of the line-of-sight speed measurements, which is the result of both the physical effects and of the algorithmic postprocessing (e.g. for interpreting the interference patterns observed with the detector). This introduces many factors related to very specific technological choices in the sensor design. These factors shall be considered when modelling a very specific system, but would introduce too many parameters for the present study. As a consequence, the sensor model that is used here represents the relative variations due to the considered design parameters in addition to an absolute measurement error level that was estimated based on available data and results of previous works. The present study provides an insight into the kind of trade-off that has to be made between the design parameters rather than a particular "best" sensor configuration.

\subsection{Results of the Sensitivity Study}

The major effect of the wake vortex disturbance on the aircraft response during a wake vortex encounter with small lateral encounter angles between $5^{\circ}$ and $30^{\circ}$, as 
they are considered here, is a rolling motion. The reduction of the maximum absolute bank angle reached during the wake vortex encounter can thus provide an indication on the overall effectiveness of the wake impact alleviation control system. It has to be kept in mind that the WIAC only generates control commands if the OWI has identified a wake vortex. As shown in Fig. 2, there are two possibilities why the OWI might provide no output. The first reason is that the OWI is not executed because the measurement noise

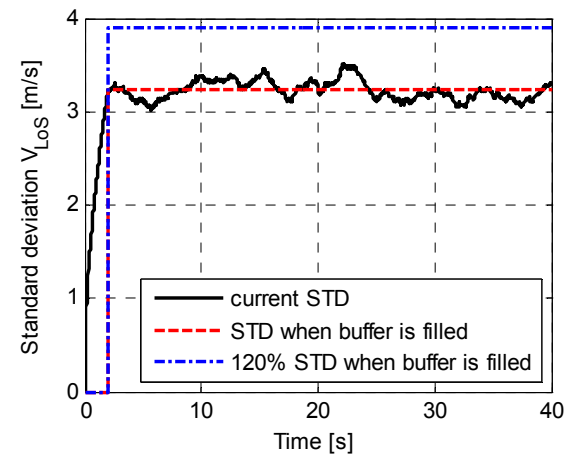

Fig. 9 Wake vortex encounter with very high measurement noise: no activation.

is very high, causing the wind velocities resulting from the wake vortex to disappear in the background noise. The second reason is that the identified wake vortex parameters are rejected by the plausibility check (cf. Section 2.1.4).

Fig. 9 exemplarily shows a wake vortex encounter during which the standard deviation of the LoS velocity of all measurement points stored in the data buffer is very high. In this case the LiDAR sensor has such a measurement noise that the standard deviation does not significantly increase when the vortex is contained in the data buffer. The threshold of $120 \%$ of the initial standard deviation of the LoS velocities is never reached and therefore the OWI is not executed during the whole simulation.

Fig. 10 illustrates the correlation between the measurement noise level, the number of plausible OWI results, and the measurement point densities. The measurement point density is defined as the number of measurement points stored in the data buffer (i.e. considered by the OWI) divided by the volume of the convex hull of all these measurement locations. The measurement noise level is assumed to be equal to the initial standard deviation of the

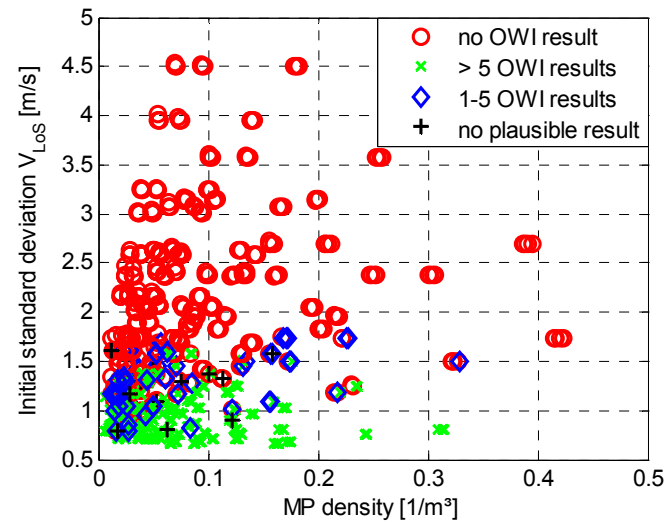

Fig. 10 Influence of initial standard deviation on execution of OWI algorithm

LoS velocities (i.e. equal to reference measurement noise as used in the activation criterion). For visibility purposes Fig. 10 only displays LiDAR settings with a blur depth of $15 \mathrm{~m}$. Red circles indicate that the optimization was not executed because the peaks of the standard deviation due to the wake vortex never reached the threshold of $120 \%$ of the initial standard deviation. Black + symbols mark cases 
where the optimization was executed (eventually several times) but none of the results ever passed the plausibility check (i.e. wake impact alleviation was never active). Blue diamonds symbolize wake vortex encounters with at least one and at most five valid OWI results and green crosses mark wake vortex encounters where more than 5 OWI results were valid and used for the wake impact alleviation during the encounter. It can be noticed that if the initial standard deviation of the considered LoS velocities is low enough (approximately $1 \mathrm{~m} / \mathrm{s}$ and below) the wake vortex can be detected and the OWI usually finds valid results. In most of the cases more than five OWI results are available. But in some cases, with less favorable LiDAR characteristics, the OWI only provides 1 to 4 results during the encounter. Furthermore, there are cases in which the OWI result does not pass the plausibility check. When the measurement noise (i.e. the initial standard deviation) increases the OWI is less often executed and even when executed the plausibility check is failed more often. On average the red circles are located above the blue diamonds and these in turn above the green crosses. The boundaries are not clearly separated because more parameters than just the noise are varied in the different cases causing different SNR for the same noise level. If the measurement noise gets very large and the initial standard deviation of the stored LoS velocities is larger than $2 \mathrm{~m} / \mathrm{s}$ the wake identification algorithm is never executed. In the majority of the investigated cases the SNR levels were too low and the activation threshold was never reached. As a consequence, Fig. 10 might give the impression that the presented wake impact alleviation system is not useful for a wake vortex encounter because it is rarely active. But it has to be kept in mind that the main cause for not executing the WIAC of the wake is the currently used activation criterion. An improved criterion should be developed and used in future studies. For LiDAR settings with a blur depth of $30 \mathrm{~m}$, cases not shown in Fig. 10, the general observations are the same. To evaluate the behavior of the OWI and the WIAC for different sensor characteristics, in the following, only those cases will be considered in which the sensor settings allowed at least one valid OWI.

As the control commands are directly derived from the identified wake vortex disturbance, it is essential to get an adequately identified wake vortex model from which the wake-induced moments can be correctly derived. Fig. 11 shows the average deviation between the rolling moment induced by the actual wake in the flight dynamic simulation and the detected rolling moment on the basis of the wake identification for the different sensor settings. This average deviation can be used as an



Fig. 11 Mean absolute error of detected compared to actually occurring wake-induced rolling moment 
identification quality index that focuses on the most important degree of freedom (roll) and considers at each point in time only the wind field errors that are currently relevant for the aircraft roll motion. It can be noticed that, apart from one outlier for a $10^{\circ}$ lateral encounter, an increase of measurement point density leads to a reduction of the error of the detected moment. The aforementioned outlier represents a case in which the OWI has found a result of bad quality but that just passed the validity check. The WIAC version applied here has no forgetting factor (i.e. the WIAC continues to use the last valid OWI result until the wake vortex was left behind the aircraft) and thus used this bad OWI result over a long time. Even though this was an unlikely case, which occurred only once in 18432 simulations, both the OWI validity check and the WIAC implementation can and should certainly be improved. The outlier case is excluded from the subsequent analysis.

An increase in measurement point density leads to a higher spatial resolution such that its correlation with reduced moment errors was expected. With the parameters that are varied there are two main possibilities to increase the density: reducing the size of the domain (e.g. with a smaller field-of-view and by reducing the minimum range) and increasing either the number of LoS directions or the number of points along each LoS. All these ways of increasing the density except for the reduction of the minimum measurement range have a negative influence on the measurement noise.

Increasing the noise level degrades the OWIDIA performance; however the positive effects of an increased spatial resolution and/or a reduction of the minimum range seem to dominate the negative effects due to the other parameters (e.g. number of MP per axis, reduction of measurement depth or increase of full scan update rate). It would be interesting to pursue the parameter variation study in that particular direction. The absolute errors between the detected and the actual wakeinduced rolling moment are larger for the smaller encounter angles. This is due to the fact that the wake-induced moments are smaller and that they are acting during a shorter time if the encounter angle increases. The relative errors (not shown here) are similar between the various encounter angles.

Fig. 12 shows the relative reduction of the maximum bank angle due to the OWIDIA system with respect to the maximum bank angle occurring without wake impact alleviation for different encounter angles and LiDAR settings. During all encounters there are no pilot inputs and the autopilot is not engaged. The only active control is an Airbus A320-like Normal Law. This law is not the original Airbus Normal Law but should behave very similarly. The upper three subplots show the variations of the encounter angle $\Psi_{\mathrm{WV}}$ and of some of the LiDAR settings for the set of simulation included in Fig. 12 (cf. Table 1). Two more parameters (blur depth and full scan update rate) are coded by different colors in the lower two plots. These two plots show the relative bank angle reduction for 60 and $90 \mathrm{~m}$ minimum range respectively. Each + sign corresponds to a single simulation. A relative value of 0.6 means that a reduction of $40 \%$ was achieved compared to the same encounter without OWIDIA. The results are split into categories labeled by letters and are visually separated by dashed vertical lines. Within each category all 
LiDAR parameters but the color-coded ones (blur depth and full scan update rate) stay the same and the wake encounter angle is varied. For readability reasons the $\Psi_{\mathrm{WV}}=30^{\circ}$ cases are not shown. In these cases the relative bank angle reduction is lower on average but very robust. However, the absolute values of the bank angle are so small that the $30^{\circ}$ encounter is practically much less relevant.

Simulations with $120 \mathrm{~m}$ minimum range are not displayed in Fig. 12 because the study revealed that these LiDAR settings are not useful for the current OWIDIA implementation. In most $120 \mathrm{~m}$ range cases the noise level was too high to allow a meaningful interpretation of the results obtained in these cases. Moreover, it also turned out that a timeframe of $2 \mathrm{~s}$ to store measurements in the data buffer is too short, especially for the smallest encounter angle of $5^{\circ}$ and large LiDAR scan angles of $30^{\circ}$ or $40^{\circ}$. In this case, the measurement points are located beyond the wake vortex when the aircraft comes close to the encounter. Hence an analysis of the $120 \mathrm{~m}$ minimum range LiDAR would only make sense if the simulation was conducted with an improved OWIDIA, e.g. with a larger buffer size and an improved activation criterion for high noise levels.

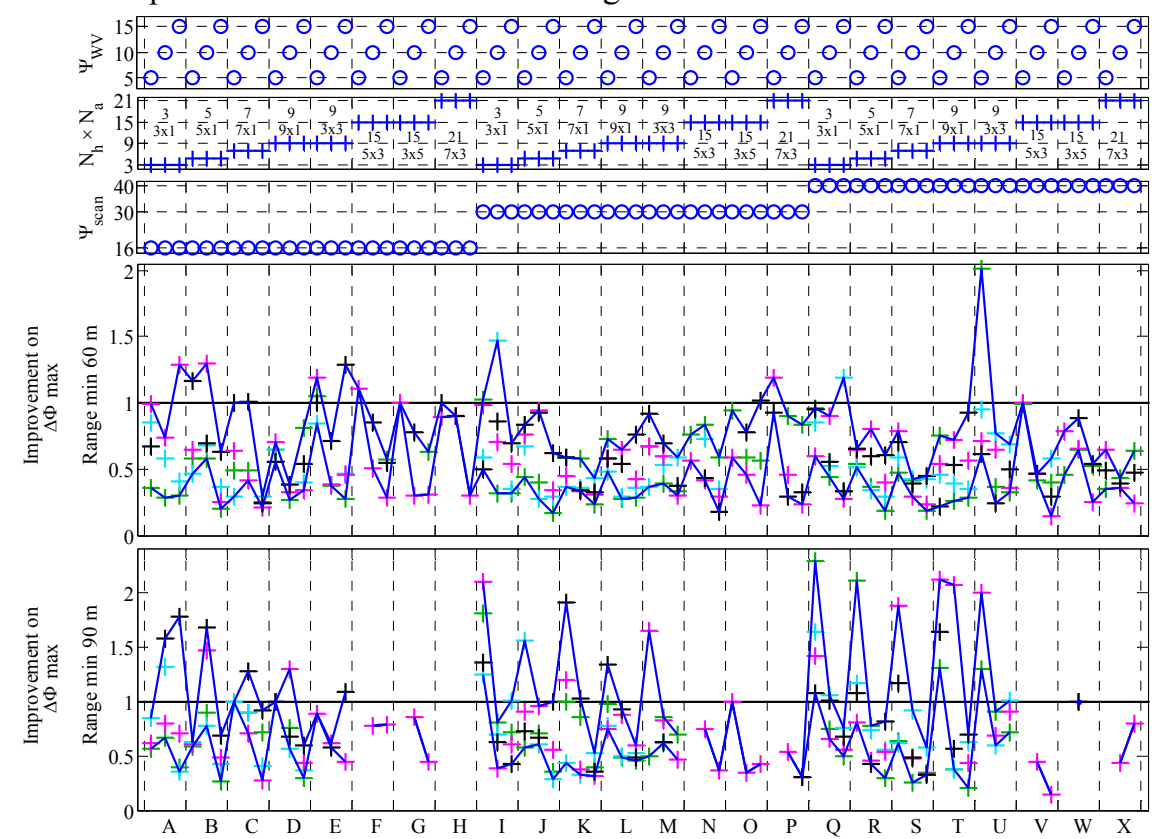

Fig. 12 Alleviation performance for different encounter angles and LiDAR settings ( + blur depth $15 \mathrm{~m}$ and full scan update rate $5 \mathrm{~Hz} \mid+15 \mathrm{~m}$ and $10 \mathrm{~Hz} \mid+30 \mathrm{~m}$ and $5 \mathrm{~Hz} \mid+30 \mathrm{~m}$ and $10 \mathrm{~Hz}$ )

Fig. 12 illustrates that some simulations show an increase of the maximum bank angle: $\Delta \Phi \max >1$, i.e. above the horizontal black line. The majority, however, showed significant improvements thanks to the OWIDIA system.

On average, LiDAR settings with a minimum range of $60 \mathrm{~m}$ provide better results than those with $90 \mathrm{~m}$ minimum range. For $60 \mathrm{~m}$ minimum range the best re- 
sults are obtained for products $\mathrm{N}_{\mathrm{h}} \times \mathrm{N}_{\mathrm{a}}$ of $7(7 \mathrm{x} 1)$ as well as the neighboring categories $(5 \times 1$ and $9 \times 1)$. This applies to all three scan angles $\Psi_{\text {scan. }}$. It results from the fact that the short minimum range and a reasonable number of measurement points provide a good trade-off between measurement noise and spatial resolution. For both 60 and $90 \mathrm{~m}$ cases and the same encounter angle $\Psi_{\mathrm{wV}}$, the vertical spread between different blur depths and full scan update rates (i.e. the different colored + signs) is sometimes very large. This seems to occur for parameter combinations that are close to or beyond the boundaries of the domain in the parameter space in which the OWIDIA system performs well. The wide spreads tend to occur more often in cases with $90 \mathrm{~m}$ minimum range and at small encounter angles. Further investigation of these cases shall help improving the OWIDIA system.

Overall, the best wake impact alleviation and most robust (against blur depth and full screen update rate) OWIDIA performance can be found in category $\mathrm{K}$ with $60 \mathrm{~m}$ range, i.e. $\Psi_{\text {scan }}=30^{\circ}, \mathrm{N}_{\mathrm{h}}=7, \mathrm{~N}_{\mathrm{a}}=1$. Categories $\mathrm{D}, \mathrm{L}, \mathrm{N}, \mathrm{R}$ and $\mathrm{S}$ with $60 \mathrm{~m}$ range also exhibit very good results for all blur depth and full screen update rate values. Further categories provide good alleviation results for some LiDAR settings and bad results in a few cases. For the blur depth and full scan update rate, no clear trend was found regarding their influence on the alleviation performance.

All in all, the OWIDIA system can reduce the maximum bank angle during a typical wake vortex encounter by $50-60 \%$ on average and up to $80 \%$ in some cases. The application of the system with appropriate LiDAR settings would allow a huge gain in terms of safety. This level of wake impact alleviation performance could support the reduction of wake vortex based separation minima for following aircraft equipped with the OWIDIA system.

\section{Summary}

A first evaluation of the complete wake impact alleviation system was presented. It uses an explicit wake identification module based on Doppler LiDAR measurements. The cross-dependencies between the modules (sensor, OWI, WIAC) are very complex and the derivation of an adequate requirement set for each module is very challenging. The overall system can only be evaluated using an environment that takes all the cross-dependencies as well as a full flight dynamics model (including flight control laws) into account. Such an environment was developed and used in this work. The main performance driver for the complete wake impact alleviation system is the LiDAR sensor. The purpose of the presented sensitivity study was to provide first insights into the domains of adequate parameter combinations. Future studies shall further refine these first results. The present study has already provided very valuable information on the system behavior and performance. It also indicates which parts of the system should be enhanced.

Parameter combinations leading to a good compromise between measurement noise and spatial resolution seem to provide the most robust performances. How- 
ever, it must be kept in mind that the applied simplified activation criteria filtered out a significant number of cases with high spatial resolution, even though these cases might have led to good impact alleviation performance. Consequently, the observed trend might have been biased through the statistical differences between the compared populations. This part of the parameter space should also be investigated in future studies, which requires an improved activation criterion. Furthermore, the behavior of the system in presence of old and deformed vortices as well as regular atmospheric turbulence should also be investigated.

Overall, a wake alleviation performance of about $60 \%$ (in terms of maximum bank angle reduction) seems achievable with the proposed system and various sets of a priori realistic sensor characteristics.

\section{References}

${ }^{1}$ TSB, "Encounter with Wake Turbulence Air Canada Airbus A319-114 C-GBHZ Washington State, USA 10 January 2008", Aviation Investigation Report A08W0007, April 8, 2010.

${ }^{2}$ NTSB, Brief of Accident LAX99LA291, Washington, D.C. May 17, 2001.

${ }^{3}$ ATSB, "Wake turbulence event Sydney Airport, NSW 3 November 2008,", Aviation Occurrence Investigation AO-2008-077, November 2009, ISBN 978-1-74251-009-5.

${ }^{4}$ NTSB, "In-Flight Separation of Vertical Stabilizer American Airlines Flight 587 Airbus Industrie A300-605R", N14053 Belle Harbor, New York November 12, 2001, NTSB/AAR-04/04, Washington, D.C., 2004.

${ }^{5}$ NTSB, Brief of Accident LAX94FA073, Washington, D.C. October 06, 1994.

${ }^{6}$ Looye, G., Lombaerts, T., Kier, T., "Design and Flight Testing of Feedback Control Laws", Research Report DLR-FB-2012-02, The DLR Project Wetter \& Fliegen, p. 162-170, 2012.

${ }^{7}$ Rafi, M., Steck, J., "Response and Recovery of an MRAC Advanced Flight Control System to Wake Vortex Encounters”, AIAA Infotech@ Aerospace Conference, Boston, August 2013.

${ }^{8}$ Schwarz, C., Hahn, K.-U., "Automated Pilot Assistance for Wake Vortex Encounters", CEAS, Berlin, Germany, 10 - 13 September 2006.

${ }^{9}$ Hahn, K.-U., Fischenberg, D., Niedermeier, D., Horn, C., "Wake Encounter Flight Control Assistance Based on Forward-Looking Measurement Processing", AIAA Guidance, Navigation, and Control Conference, Toronto, Ontario Canada, 2 - 5 August 2010.

${ }^{10}$ Kloidt, S., "Beiträge zum Entwurf eines Flugregelungssystems zur Reduktion des Wirbelschleppeneinflusses (English: Contributions to the Design of a Flight Control System for the Reduction of the Wake Vortex Impact)“, Ph.D Thesis, TU Berlin, Germany, 2007.

${ }^{11}$ Ehlers, J., Fischenberg, D., Niedermeier, D., „Wake Identification Based Wake Impact Alleviation Control“, AIAA AVIATION, Atlanta, GA, US, 16-20 June 2014.

${ }^{12}$ Fischenberg, D., "Online Wake Identification Algorithms Using Forward Looking LIDAR Sensor Measurements", DLR Report IB111-2013/11, DLR German Aerospace Center, Braunschweig, Germany, February 2013.

${ }^{13}$ Fischenberg, D., "Strömungsermittlungsverfahren/Flow Determination Method/Procédé de détermination d'écoulement", Patent No. EP 2340438 B1, European Patent Office 2013.

${ }^{14}$ Jategaonkar, R. V., "Flight Vehicle System Identification: A Time Domain Methodology", Progress in Astronautics and Aeronautics, AIAA, Reston, VA, 2006.

${ }^{15}$ Burnham, D. C., Hallock, J. N., "Chicago Monoacoustic Vortex Sensing System", Vol. 4, Wale Vortex Decay, Springfield, VA, National Information Service, 1982.

${ }^{16}$ Fischenberg, D., "A method to validate wake vortex encounter models from flight test data", ICAS 2010, 27th International Congress of the Aeronautical Sciences, Nice, France, 2010. 